\title{
Les archives audio du théâtre. Enjeux ontologiques des captations de la représentation sur bande magnétique
}

The Theatre's Audio Archives: Ontological Issues Regarding the Capture of Performance on Magnetic Tape

Jean-Marc Larrue

\section{OpenEdition}

\section{Journals}

Édition électronique

URL : https://journals.openedition.org/rsl/1598

DOI : $10.4000 /$ rsl. 1598

ISSN : 2271-6246

Éditeur

Éditions Rue d'Ulm

\section{Référence électronique}

Jean-Marc Larrue, «Les archives audio du théâtre. Enjeux ontologiques des captations de la représentation sur bande magnétique », Revue Sciences/Lettres [En ligne], 6 | 2019, mis en ligne le 10 décembre 2018, consulté le 30 juillet 2021. URL : http://journals.openedition.org/rsl/1598; DOI :

https://doi.org/10.4000/rsl.1598

Ce document a été généré automatiquement le 30 juillet 2021.

(c) Revue Sciences/Lettres 


\title{
Les archives audio du théâtre. Enjeux ontologiques des captations de la représentation sur bande magnétique
}

\author{
The Theatre's Audio Archives: Ontological Issues Regarding the Capture of \\ Performance on Magnetic Tape
}

Jean-Marc Larrue

1 Cet article porte sur un projet de recherche ${ }^{1}$ qui a pour objet la bande magnétique audio au théâtre. Ce projet fait partie d'une programmation plus vaste dont l'objectif est de préciser l'apport de la bande magnétique audio à diverses pratiques médiatiques et artistiques dont, en premier lieu, le théâtre et les arts vivants, mais aussi la musique, le disque, la radio et le cinéma où, par elle, a éclos la technique du cinéma direct. Mais la bande magnétique a aussi pénétré le champ des arts visuels, grâce à une pratique émergeant avec succès des années 1960, l'installation. Les concepteurs de celle-ci recouraient en effet régulièrement à des bandes magnétiques pour baigner leurs œuvres dans un univers sonore singulier'2 ${ }^{2}$. Si l'installation, au moins à ses débuts, était liée aux néo-avant-gardes et restait limitée à un cercle restreint d'amateurs, tel n'était pas le cas des spectacles «son et lumière » qui fleurissaient à la même époque et attiraient des foules considérables, de tout âge et de tout milieu. Or, on imagine mal ce formidable succès sans la souplesse et les qualités de captation et de reproduction des bandes magnétiques audio ${ }^{3}$.

2 L'influence de la bande magnétique audio a ainsi touché un très grand nombre de pratiques dont elle a modifié l'audibilité. Comme le terme "transmédial » s'applique, selon l'acception courante, à la diffusion de "contenu médiatique " à travers différentes plateformes (ou technologies, ou supports, ou médias), on serait tenté de qualifier de «transmédiatique » l'action tentaculaire de cette bande magnétique audio qui est bien plus qu'une "technologie de livraison» ou qu'un "média technique ${ }^{4}$ ». D'ailleurs, si l'on vivait encore à l'époque où le terme média faisait consensus, on 
pourrait affirmer que cette bande est un média à part entière, c'est-à-dire, pour reprendre la définition classique d'Eliseo Verón, "un ensemble constitué par une technologie PLUS ${ }^{5}$ les pratiques sociales de production et d'appropriation de cette technologie ${ }^{6} »$.

Mais la bande magnétique n'est pas qu'audio et son apport dépasse le strict domaine du son. Rappelons, pour mémoire, que la bande magnétique a servi de support aux captations vidéographiques avant l'avènement du numérique, et que les premières saisies de données informatiques ont été enregistrées sur des bandes magnétiques. Le lien entre l'analogique et le numérique est d'ailleurs plus complexe que l'image qu'on en a généralement, fondée sur des oppositions simplistes ou une successivité plus que discutable. L'industrie a même produit pendant quelques années des magnétophones (à ruban) numériques. Mais dans le cadre de ce vaste projet de recherche, il a été convenu de focaliser l'attention sur la bande magnétique audio et sur son apport à la dimension sonore des diverses pratiques artistiques et médiatiques retenues. En sont donc exclus les usages non sonores de la bande magnétique, en dépit des transformations majeures qu'ils ont produites sur les socialités. Le texte qui suit est encore plus restreint puisqu'il se limite à la bande magnétique audio au théâtre.

4 Si les études techniques sur la bande magnétique et sur l'histoire des appareils - qui permettaient de fixer les sons sur cette bande et de les reproduire ensuite - sont abondantes, les données dont nous disposons sur le rôle qu'elle a joué dans le développement du son de la représentation théâtrale et dans l'étude de celui-ci (grâce aux enregistrements que nous en avons conservés) sont encore fragmentaires et limitées. Pourtant, tout indique que ce rôle a été considérable.

On situe généralement les premières utilisations de la bande magnétique audio au théâtre ${ }^{7}$ dans la deuxième moitié des années 1950, soit plus de vingt ans après la commercialisation - en 1937 en Allemagne - de cet appareil enregistreur nommé "Magnetophon", le magnétophone à ruban, dont Hitler et les nazis ont fait couramment usage durant la Seconde Guerre mondiale dans leurs opérations de propagande. L'opinion dominante aujourd'hui veut que le recours à la bande magnétique par les gens de théâtre ait été inspiré par la bande-son du cinéma. Cette dernière, on le sait, était en fait et paradoxalement depuis la fin des années 1920 une bande optique. La bande-son du théâtre serait donc une "remédiation ", au sens de Bolter et $\mathrm{Grusin}^{8}$, de la bande-son cinématographique, qui aurait au passage migré d'une plateforme à une autre. Si elle repose sur des constats indiscutables - par exemple, le fait que certains des premiers « concepteurs » de son au théâtre venaient du cinéma où ils avaient produit des bandes-son -, cette opinion est cependant réductrice. D'une part, il existait, avant le magnétophone, une longue tradition d'accompagnement sonore - musique, bruitage - du dialogue sur les scènes de théâtre ; d'autre part, tout indique que des facteurs externes au cinéma ont également joué dans l'émergence de la bande-son théâtrale. À cet égard, il ne faudrait pas sous-estimer l'effet des explorations électroacoustiques et, également, le rôle des artisans de la radio, en France comme à l'étranger, dans le développement d'environnements sonores de plus en plus complexes qui accompagnaient et englobaient la parole à la radio'.

6 Le projet de recherche sur la bande magnétique audio que nous menons relève en partie de l'archéologie des médias, mais l'essentiel est d'un autre ordre. L'hypothèse que nous défendons est simple : la bande magnétique audio aurait radicalement changé l'auralité théâtrale - ce qu'on entend au théâtre, comment on l'entend et ce qu'on en 
éprouve - et la fabrique du son de la représentation. Ce changement aurait été si déterminant et si irréversible, si chargé de nouveauté aussi, qu'on peut qualifier ces trois à quatre décennies marquées par l'usage du magnétophone à ruban de période « analogique » du théâtre, une période qui, dans l'histoire du son moderne du théâtre, succéderait à l'ère électromécanique ${ }^{10}$. Il y aurait donc deux époques du son moderne du théâtre, qui débute avec l'avènement des technologies de reproduction du son au tournant des années $1880^{11}$, l'ère électromécanique (1880-1950) et l'ère magnétonumérique (1950 à aujourd'hui); cette dernière serait elle-même divisée en deux périodes, la période analogique (1950-1990) et la période numérique (de 1990 à aujourd'hui).

7 Mais la bande magnétique audio n'a pas seulement révolutionné le son du théâtre, elle est aussi vite devenue, à l'instar des phonogrammes des premiers temps, la précieuse archive d'un moment - l'évènement théâtral - réputé éphémère. Si l'apport de cette archive à la recherche est inestimable, son existence soulève des questions de fond, à la fois sur les pratiques archivistiques et sur la pensée théâtrale.

\section{La période analogique de l'ère magnéto-numérique : perspectives historiques et théoriques}

8 Il y a une dizaine d'années, quand a commencé à émerger ce champ qu'on nomme à présent les études sonores en théâtre, on avait raison d'affirmer que la réalité sonore de la représentation avait jusque-là peu soulevé l'intérêt des chercheurs. Dix ans plus tard, s'il est évident qu'il reste encore beaucoup à faire, on ne peut plus parler d'oubli du son. Un travail colossal de rattrapage a été abattu. En quelques années, plus de 150 textes ont été produits sur la question dont la quarantaine d'articles publiés en 2016 dans les numéros 56 et 57 de la revue L'Annuaire théâtral et dans l'ouvrage collectif Le Son du théâtre (XIXé-XXI siècle). Histoire intermédiale d'un lieu d'écoute moderne ${ }^{12}$. À cela, il faut ajouter la soixantaine de textes publiés dans les numéros 197, 199 et 201 de la revue Théâtre/Public en 2010 et 2011. Or, même au sein de ce corpus récent, qui témoigne de l'existence et du dynamisme des études sonores en théâtre et qui dresse un portrait impressionnant des innovations constantes survenues dans le son de la représentation depuis la fin du XIX $X^{e}$ siècle, la bande magnétique est à peu près ignorée. Si certains articles abordent le sujet de manière indirecte, le seul qui en traite spécifiquement est celui que Guillaume Trivulce consacre à la bande-son dans le numéro 199 de Théâtre/Public en $2010^{13}$. Les choses changent cependant depuis 2014 grâce au projet ECHO qui a précisément pour objectif de mettre au jour les transformations majeures vécues au cours de cette période, entre autres dans le domaine de la voix parlée scénique et de l'auralité théâtrale, et s'est intéressé au magnétophone de trois façons différentes: en réalisant le fichage méthodique ( $\mathrm{du}$ protocole d'écoute à une triple description du contenu) d'une centaine d'enregistrements des années 1950-1990 (captations de représentations ou matériaux sonores des spectacles) dont le support était presque toujours la bande magnétique ${ }^{14}$; en étudiant des spectacles où le magnétophone a joué un rôle important ${ }^{15}$; en collectant des témoignages de régisseurs et ingénieurs son de différentes générations ${ }^{16}$.

9 Aussi, de même qu'on déplorait, il y a une décennie, alors qu'émergeaient les études sonores en théâtre, l'« oubli » du son du théâtre, on réagit à présent à l'oubli de la 
bande magnétique à l'intérieur même de ce champ d'étude. Cet oubli est étonnant et ne l'est pas.

10 Commençons par la non-surprise. Les travaux menés en études sonores en théâtre, avant le projet $\mathrm{ECHO}$, se concentrent sur deux périodes: celle durant laquelle les technologies de reproduction du son entrent dans la fabrique du son théâtral, soit de la fin du XIX ${ }^{e}$ siècle au tournant des années 1950, et la période actuelle, correspondant à l'extrême contemporain, où se multiplient les études d'œuvres récentes jugées marquantes par leur dimension sonore. Cette focalisation n'était pas le fruit du hasard, comme je vais l'indiquer plus loin, mais elle a eu pour effet de laisser un trou béant de quatre décennies environ, qui correspondent sensiblement à cette période analogique oubliée.

11 Poursuivons avec l'étonnement: tout indique que la bande magnétique et, donc, le magnétophone, ont radicalement transformé l'auralité théâtrale et la fabrique des sons reproduits. Le magnétophone a ainsi grandement facilité la création et la diffusion de sons nouveaux. John Cage, qui a beaucoup composé avec des magnétophones, parle dans sans son livre Silence de la bande magnétique - the Tape - comme d'un instrument de musique au potentiel révolutionnaire et infini. Cet instrument a été en effet un facteur déterminant dans l'instauration d'une véritable pratique du montage sonore qui, jusque-là, était resté assez rudimentaire. Le vaste chantier de recherche consacré aux relevés de mise en scène de l'Association de la régie théâtrale (ART), mené de 2012 et 2015 à la Bibliothèque historique de la ville de Paris ${ }^{17}$ (BHVP), a permis de préciser la nature de ces montages avant le magnétophone ${ }^{18}$. Essentiellement, il s'agissait de passer manuellement et de façon aussi précise que possible - au bon moment et au bon endroit sur le disque - de sillons prédéterminés d'un disque installé sur un premier gramophone/phonographe à ceux d'un disque installé sur un second appareil. Certaines installations, particulièrement sophistiquées, pouvaient comprendre davantage de gramophones/phonographes mais, quels que furent le nombre d'appareils utilisés et la dextérité des techniciens mobilisés, ces montages phonographiques restaient limités dans leurs effets. Ce n'est pas le cas du montage sonore magnétique qui, en plus de multiplier les possibilités de structuration de l'univers sonore des spectacles et les modalités de passage d'une section sonore à l'autre, permettait une diversification sans précédent des sons reproduits par la combinaison de sons artificiels, de sons musicaux et de sons naturels saisis dans l'environnement. Tout cela a progressivement - puisque la transformation a été lente et irréversiblement changé la fabrique du son du spectacle tout en participant de l'autonomisation de ses artisans. La fonction de designer ou concepteur sonore commence à se définir véritablement avec le magnétophone.

12 L'analyse des bandes-son théatrales révèle à la fois l'écart qui séparait le montage magnétique du montage phonographique et la proximité de celui-là avec le montage réalisé à l'aide de technologies numériques - ou montage numérique. L'analyse de créations sonores sur bande magnétique porte même à penser que les technologies numériques n'ont fait que faciliter, accélérer et amplifier les procédés inventés et développés durant la période analogique. Pour paraphraser Milad Doueihi ${ }^{19}$, on pourrait dire que s'il y a bien eu une "grande conversion numérique », la véritable révolution dans le domaine du son au théâtre a été magnétique. Cela va dans le sens de ce qu'affirment des concepteurs sonores marquants de la scène théâtrale qui ont également connu l'époque des magnétophones tels le Français Daniel Deshays ou 
l'Américain Scott Gibbons (concepteur sonore lié à Romeo Castellucci). Mais ce qui apparaît de plus en plus à présent comme une évidence va à l'encontre de l'idée qui dominait le champ des études sonores il y a dix ans et qui explique l'intérêt des chercheurs pour la période 1880-1950 et la période qui va des années 1990 à aujourd'hui. Par simple décalque d'une périodisation qui prévaut encore dans le champ des études médiatiques, la modernité est née d'une révolution, l'avènement des technologies électriques, et aurait été relancée, environ un siècle plus tard, par une nouvelle révolution, celle du numérique. Ce balisage, bruyamment relayé par l'industrie des technologies de reproduction du son et de l'image, doit être nuancé. Dans le cas du son au théâtre, il y a lieu de le reconfigurer : la seconde révolution aurait été magnétique.

13 La bande magnétique audio a eu d'autres impacts majeurs, aujourd'hui si naturalisés qu'il n'est pas inutile de les rappeler. Elle a en effet bouleversé les conditions de la diffusion sonore, en entraînant - ou du moins en étant le principal facteur de - la sonorisation massive des salles à partir des années 1960. Cette transformation généralisée du bâti théâtral s'est faite elle aussi progressivement. Jusqu'aux recherches toutes récentes menées dans le cadre des travaux du groupe ECHO, on disposait de peu de données sur cette modification majeure apportée aux salles, grâce à laquelle le son « reproduit » émis à partir d'une console de son arrive à des haut-parleurs fixes à travers un système d'amplification. Une nouvelle auralité naissait, composée - sauf exception ${ }^{20}$ - de la voix des acteurs (et des bruits de leur jeu) entendue en direct et des sons reproduits diffusés par les haut-parleurs ${ }^{21}$.

\section{La bande magnétique audio en tant qu'archive : enjeux et défis}

14 Une fois le cycle des spectacles terminé, les bandes-son du théâtre deviennent d'inestimables sources d'information pour la recherche en études sonores: nature, variété et évolution des sons produits, diversité des transitions, extension de l'accompagnement sonore, etc. Mais la bande magnétique audio n'a pas seulement servi de bande-son, elle a aussi permis une pratique documentaire inédite, la captation "directe» de la représentation théâtrale. S'il existe une longue tradition d'enregistrements cinématographiques, phonographiques ou radiophoniques du spectacle théatral - ou d'extraits de spectacle -, celle-ci repose sur des opérations complexes qui imposent de préformater la captation en vue de sa diffusion subséquente - ce qui relève de ce que Grusin appelle la "prémédiation ${ }^{22}$ " - ou de transformer ( $a$ posteriori) la matière enregistrée dans le but de la conformer au média de diffusion concerné (disque, radio, film), ce qui relève du principe général de remédiation avec tous les phénomènes d'effacement et d'opacification que cela entraîne inévitablement.

15 Les captations "directes » sur bande magnétique audio dont il est question ici sont d'une autre nature, elles n'ont habituellement pas de fonction autre qu'archivistique mais, paradoxalement, elles étaient jusqu'ici rarement traitées comme telles. Réalisées à l'aide d'un magnétophone à partir de la régie de son ou par une personne située dans le public (un spectateur ordinaire, un critique), elles ne font l'objet d'aucun montage et demeurent à l'état brut (à la différence des bandes, presque identiques, enregistrées dans une perspective radiophonique ou discographique). Si, évidemment, le fait d'enregistrer impose de se plier à certaines contraintes techniques - ne serait-ce que la 
capacité limitée d'enregistrement (en durée) des bobines qui est généralement inférieure à la durée des spectacles, ce qui nécessite des manipulations durant lesquelles rien n'est capté -, il n'y a à proprement parler ni prémédiation, ni superposition de médialités, ni soumission de l'une (la médialité de la scène) à l'autre (celle de la bande) dans l'opération d'enregistrement. Insistons sur ce fait: si les contraintes et les limites de la technologie magnétique, ses «bruits", les inévitables transformations, voire distorsions, qu'elle produit - à commencer par une indifférenciation spatiale de la source ou des sources à partir desquelles les sons sont captés (à l'aide d'un micro unique, le plus souvent) - constituent des écarts par rapport au son original de la représentation, les enregistrements qu'elle produit sont ce qui nous en rapproche le plus. Disons-le autrement: la bande magnétique audio nous permet d'approcher l'expérience spectatorielle passée comme aucune autre technologie ne l'avait fait avant elle. Ce que révèlent ces enregistrements est étonnant, comme l'illustrent les deux extraits tirés des archives sonores de la Théâtrothèque de l'Université de Montréal, dont il est question plus loin. Les données qu'on y trouve, tant sur le spectacle que sur le public, sont de nature à bouleverser certains faits établis de l'histoire du théâtre. Mais ce qui est plus étonnant encore c'est que, malgré cette remarquable valeur heuristique, les captations sonores directes demeurent très négligées et leur statut incertain, en tant qu'archives, soulève des questions de fond qui touchent à la dimension ontologique du théâtre lui-même.

Le geste d'archiver - on devrait dire les gestes d'archiver, tant les processus d'archivage impliquent une vaste agentivité -, est tout sauf neutre. Non seulement estil pris dans des usages de la mémoire collective et individuelle, dans des formes d'institution du passé, dans des pratiques de conservation et dans des techniques de transmission et donc de déplacement, mais il est aussi le résultat de décisions politiques, de rapports de pouvoir et d'enjeux sociaux aussi bien que symboliques. Il faut insister sur cet aspect : tout archivage est fondé sur des régimes d'autorité. S'il est vrai qu'archiver revient à autoriser un document à s'inscrire dans un présent perpétuel, on doit admettre que les captations directes dont il est question ici ont un statut archivistique plus que limité. Contrairement aux documents visuels et imprimés (les textes), ces enregistrements revêtent habituellement si peu de valeur aux yeux mêmes des personnes qui les ont produits qu'ils n'ont, dans la plupart des cas, fait l'objet d'aucun classement ni d'aucune mesure assurant leur conservation - et encore moins leur transmission ou diffusion - avant d'être éventuellement (et trop rarement) pris en charge par des institutions spécialisées. Quand on est invité à examiner les fonds d'archives de théâtres ou de compagnies de théâtre, comme cela m'arrive fréquemment à Montréal, on découvre souvent des tas de bandes magnétiques empilées pêle-mêle dans des boîtes non identifiées rongées par l'humidité. Personne ne sait d'où elles viennent et les indications qu'on trouve parfois sur les bobines ne sont guère éclairantes. On se demande même par quelle négligence providentielle elles n'ont pas été tout simplement mises au rebut. Le fait que le milieu, pour dire les choses simplement, ait accordé si peu d'importance à ces archives et en décourage la diffusion - ce qui est également vrai des captations vidéo qui sont devenues la norme dans la pratique actuelle des producteurs - est très révélateur. Il y a en effet, latente, une "non-autorisation" systémique, généralisée, qu'on ne peut expliquer que par des motifs idéologiques. Ceux-ci reposent sur une conception essentialiste du théâtre qui, contre toute attente, perdure encore. Elle découle d'une sacralisation du lien « naturel » - c'est-à-dire non technologisé - de l'acteur au spectateur, qui s'est imposée 
au cours du $\mathrm{Xx}^{\mathrm{e}}$ siècle $^{23}$, et d'une profonde méfiance à l'égard de toute médiation technologique de la représentation qui, curieusement, résiste à une pratique scénique plus technologique que jamais.

Le traitement réservé à ces documents et la réticence à les valoriser ne découlent pas d'un simple réflexe technophobe, puisque toutes les autres archives de la représentation ou qui sont liées à elle - les textes, les costumes, les accessoires de décors et, plus encore, les photographies - sont, d'une façon ou d'une autre, des produits technologiques. Ils tiennent plutôt à la nature spécifique de ces archives qui ont pour singularité de fournir une trace de la représentation dans sa durée (comme les captations vidéo actuelles). C'est visiblement cette qualité temporelle spécifique qui cause problème. Déjà en 1993, dans un essai percutant qui allait déclencher une longue polémique $^{24}$, Peggy Phelan excluait toute possibilité de reproduction de la représentation : "Dans la mesure où la représentation tente d'entrer dans l'économie de la reproduction, elle trahit et diminue la promesse de sa propre ontologie ${ }^{25} »$. Cette idée de "trahison", qui n'est pas sans évoquer la perte d'aura de l'œuvre d'art que décrit Benjamin dans son célèbre essai sur les effets de la reproductibilité technique en $\operatorname{art}^{26}$, fut reprise et explicitée par Gay McAuley, dans le sillage de l'essai de Phelan.

[L]e théâtre est par nature un art du moment présent et les artistes de théâtre focalisent leurs énergies sur le présent de l'expérience vécue. La représentation ne peut être répétée et elle est justement fascinante aux yeux des acteurs comme des spectateurs précisément parce qu'elle est unique et éphémère ${ }^{27}$.

Phelan et McAuley perpétuaient ainsi un discours identitaire, apparu dans le premier tiers $\mathrm{du} \mathrm{xx}^{\mathrm{e}}$ siècle, qui proclamait la supériorité ontologique du théâtre sur les médias électriques qui le concurrençaient (radio et cinéma, en particulier) : « La suprématie du théâtre dérive du fait qu'il est toujours "maintenant" sur scène ${ }^{28}$ ", affirmait ainsi Malcolm Cowley en 1962, au moment où se multipliaient les captations audio non radiophoniques et non phonographiques des représentations théâtrales. Cette conception ontologique se fonde sur une synthèse irréductible où l'articulation de l'éphémère, l'unicité et la non-répétitivité au concept phare de présence est présentée comme une évidence. Et, dans cette pensée simplette où la présence est le contraire du médiatisé, on peut difficilement concevoir l'éphémère autrement que dans son instantanéité, donc dans sa continuelle disparition immédiate. L'argument de la présence ainsi élargi n'est pas seulement contredit par la réalité de la scène - où les technologies et le médiatisé sont omniprésents -, il va à l'encontre du "désir de rétention ${ }^{29}$ " de l'humain auquel les archivistes s'efforcent de répondre. "Si nous ne documentons pas la représentation, elle disparaît; nous documentons la représentation pour qu'elle cesse de disparaître ${ }^{30}$ ", affirme Matthew Reason à l'encontre des thuriféraires de l'éphémère. Le théâtre "trahirait les promesses de son ontologie » s'il entrait dans « l'économie de la reproduction ». Mais quelle trahison?

On peut l'affirmer sans trop de risque d'erreur, les propagateurs de cette vision ontologique du théâtre ont détourné à des fins idéologiques une caractéristique que le théâtre partage avec d'autres pratiques. Au lieu de reconnaître que, même dans le théâtre le plus dénudé, la présence est toujours le produit de conjonctures médiatrices - qu'elle est donc médiatisée -, ils ont érigé cette présence en absolu, insistant sur sa rareté et son caractère insaisissable. Il est difficile de départager, dans leur action longue et complexe, ce qui relève du sentiment technophobe, des convictions religieuses - et de la croyance en la supériorité du naturel (divin) sur l'artificiel (humain) -, de l'affairisme, de la nostalgie, de l'angoisse ou de la simple naïveté. Mais 
rêvons un instant: quelle mine remarquable d'information nous fournirait une captation audio de la création de Hamlet vers 1600 ou du Malade imaginaire de 1673 ? À quoi ou à qui nuirait cette entrée dans "l'économie de la reproduction" ? Sûrement pas à l'histoire du théâtre. Qui serait trahi ? Personne. Et qu'est-ce qui serait trahi ? On se le demande !

Nous avons abordé les captations sonores des représentations sur bande magnétique dans une perspective théorique, en soulevant quelques questions sur la nature de ces archives et sur leur statut dans le monde du théâtre. Les indices que nous avons pu recueillir jusqu'à présent au sujet de captations sonores ou audiovisuelles au sein d'autres arts vivants portent à penser que la situation y est fort différente, le théâtre représenterait un cas particulier. C'est à cette étude comparative que sera consacrée la dernière partie de notre programmation de recherche. Pour l'heure, l'essentiel du travail consiste à écouter les captations existantes et à en démontrer l'exceptionnelle valeur documentaire pour mieux en assurer, autant que possible, la préservation. Cela, comme on vient de le voir, a des résonances profondes et met en jeu des perceptions ancrées dans le milieu depuis près d'un siècle.

\section{En guise de conclusion : écoutons...}

Pour clore cette réflexion, nous proposons une plongée dans ces captations par l'écoute de deux brefs extraits tirés de la collection des archives sonores de la Théâtrothèque de l'Université de Montréal. Centre d'archives et de recherche en théâtre, la Théâtrothèque compte près de trois cents captations audio, sur bande magnétique et cassettes, de pièces québécoises francophones réalisées par des troupes professionnelles entre 1963 et 1985.

L'analyse de ces archives vient de commencer, il en ressort déjà quelques constats. Le premier est l'absence quasi généralisée d'appui sonore au dialogue - musique, bruitage, environnement sonore - durant cette période. Il semblerait donc que, au Québec, la bande magnétique comme support de captation aurait précédé la bande magnétique comme bande-son de la représentation. Un deuxième aspect important qu'on retient de ces captations est le vieillissement du jeu. De toute évidence, la voix résonante est encore dominante jusqu'aux années 1980, on parle avec emphase, même dans les comédies et les scènes les plus réalistes. En même temps, on note des procédés aujourd'hui disparus comme l'accélération marquée du débit dans les passages comiques du dialogue. De façon générale, d'ailleurs, le débit semble plus rapide qu'aujourd'hui. Le vieillissement du jeu se double du vieillissement de la langue (québécoise) qui est vraiment frappant: expressions datées, formulations disparues, même la prononciation a changé. Un autre élément digne de mention est la réception de ces pièces telle qu'elle se manifeste de façon sonore. On relève, dans toutes les captations écoutées, une forte et étonnante présence du public.

Deux extraits d'enregistrements sonores de deux mises en scène de textes de Michel Tremblay : Les Belles-Sœurs (en 1969) et $\dot{A}$ toi, pour toujours, ta Marie-Lou (en 1979). Documents de la Théâtrothèque de Montréal, montage réalisé en 2018 par Charlotte Gagné-Dumais (12'01"). docannexe/file/1638/larrue_tremblay.mp4

Le premier extrait est composé de deux segments. Il est tiré d'un enregistrement pirate de la pièce phare du théâtre québécois, Les Belles-Sœurs de Michel Tremblay. Réalisé par 
un spectateur à l'aide d'un magnétophone à ruban et d'un micro unique, cet enregistrement date de 1969 ; il s'agit donc de la reprise du spectacle créé en 1968, avec quelques changements de distribution. Comme c'est le cas dans la très grande majorité des enregistrements sonores de cette nature, nous possédons très peu de données sur les circonstances de la captation. Dans la première partie de l'extrait, les «bellessœurs » découvrent qu'Angéline Sauvé, qu'elles pensaient irréprochable, fréquente une boîte de nuit (un club). L'épisode est monté de façon chorale. La seconde partie est la fameuse « Ode au bingo ${ }^{31}$ ».

Le second extrait date de 1979. Contrairement au précédent, celui-ci est réalisé à même la régie avec un micro placé sur scène. Il s'agit de la captation de À toi, pour toujours, ta Marie-Lou, autre pièce de Michel Tremblay. Le spectacle a été enregistré en 1979 à Louvain-la-Neuve en Belgique lors d'une tournée de la production québécoise en Europe. On peut présumer que c'est à la demande de l'organisme subventionnaire public (qui a sans doute financé la tournée) que cette archive a été réalisée. Le mode d'enregistrement explique la qualité étonnante de cette captation. L'extrait choisi correspond à l'un des moments les plus dramatiques de la pièce, alors que Léopold reproche à sa femme Marie-Lou l'absence de sexualité dans la vie du couple. Outre le jeu, le fait saillant de cet extrait est le comportement du public qui manifeste de façon très audible son opinion sur la question.

L'essentiel du travail d'analyse reste à faire, tant dans ces deux captations phares que dans les dizaines d'autres que nous comptons étudier. Le développement d'un protocole d'écoute, qui est évidemment au cœur de cette démarche, est en voie de réalisation. Il devrait non seulement permettre de rendre compte de chacune des captations et bandes-son que nous avons retenues mais aussi, et plus globalement, de mieux saisir comment l'auralité de la scène théâtrale a évolué dans cette période de rapides changements technologiques marquée par le triomphe et le déclin du magnétophone et de l'enregistrement magnétique.

N. B. Le montage sonore a été réalisé en mai 2018 par Charlotte Gagné-Dumais, doctorante et auxiliaire de recherche à la Théâtrothèque de l'Université de Montréal.

\section{NOTES}

1. Il s'agit d'un projet de recherche financé par le Conseil de recherche en sciences humaines du Canada (CRSH) pour la période 2017-2021, intitulé «La révolution magnétique et le théâtre, lieu d'écoute intermédiale de la modernité : le cas montréalais (1955-1990) ».

2. Pour une analyse détaillée de l'usage du son par les avant-gardes, voir l'essai de Mladen Ovadija, Dramaturgy of Sound in the Avant-garde and Postdramatic Theatre, Montréal et Kingston, McGill-Queen's University Press, 2013.

3. Voir infra Éric Monin, « Les techniques pionnières des premiers spectacles son et lumière ».

4. Ces deux expressions, à peu près équivalentes, sont dues respectivement à Henry Jenkins et à Lars Elleström. Elles désignent les «supports » sur lesquels repose la médiation. Jenkins affirme 
ainsi que la reproduction du son est le média, la cassette audio, par exemple, serait la technologie de livraison.

5. Souligné par l'auteur.

6. Eliseo Verón, «De l'image sémiologique aux discursivités », Hermès, Espaces publics en images, $\mathrm{n}^{\circ} 13-14,1994$, p. 51.

7. L'opéra et le musical n'ont pas encore été analysés sous cet angle mais le seront dans une phase ultérieure.

8. Voir Jay David Bolter et Richard Grusin, Remediation. Understanding New Media, Cambridge (Mass.), MIT Press, [1999] 2000.

9. Voir infra Marion Chénetier-Alev, «Les archives radiophoniques du théâtre. Du théâtre pour les aveugles à un théâtre de sourds?».

10. Cette hybridité - électricité et mécanique - s'explique par le caractère purement mécanique des premiers phonographes et gramophones (dont l'électrification ne se généralise que dans les années 1920).

11. Avec le développement presque simultané du microphone, du phonographe et du téléphone.

12. Jean-Marc Larrue et Marie-Madeleine Mervant-Roux (dir.), Le Son du théâtre (XIX ${ }^{e}$-XXI ${ }^{e}$ siècle). Histoire intermédiale d'un lieu d'écoute moderne, Paris, CNRS Éditions, 2016.

13. Guillaume Trivulce, "La "bande-son" au théâtre", Le Son du théâtre. 2. Dire l'acoustique, Théâtre/Public, n 199, printemps 2011, p. 32-35.

14. Ces fiches, qui ont servi à enrichir le catalogue de la BnF, seront réunies et consultables dans la collection ECHO de HAL. Pour des exemples d'usages de ces bandes par la recherche, voir infra les études de Joël Huthwohl, Joëlle Deniot et Brigitte Joinnault. [Lien au dossier pédagogique multimédia en ligne " Entendre le théâtre », à venir début juillet 2019] Voir aussi M.-M. MervantRoux, «Les archives sonores de L'Amante anglaise (Paris, TNP, 1969). L'enregistrement contre la mémoire ?", in M. Noonan et J. Pagès-Pindon (dir.), Marguerite Duras. Un théâtre de voix, Leyde (Pays-Bas), Brill, 2018, p. 75-91.

15. Voir infra Joël Huthwohl, « Tellur, Yllen et la stéréo. Musique, sons et voix dans Nucléa au TNP en $1952 »$.

16. Voir infra Daniel Deshays, « Régie et archivage au quotidien des représentations ».

17. Pour plus de détails sur ce fonds exceptionnel, voir http://www.regietheatrale.com/index/ index/collections.htm

18. Voir aussi Bruno Sebald et Marie-Gabrielle Soret, sur Jouvet et l'usage des disques dans les spectacles : «Les archives sonores du fonds Jouvet », Le son du théâtre. 2. Dire l'acoustique, Théâtre/ Public, nº 199, op. cit., pp. 17-27.

19. Milad Doueihi, La Grande Conversion numérique, Paris, Seuil, « La librairie du XXI ${ }^{\mathrm{e}}$ siècle », 2008.

20. Certains théâtres, à l'acoustique vraiment déficiente, amplifiaient la voix des acteurs mais c'était tout à fait exceptionnel. Pour connaitre l'une de ces exceptions, dont on ignorait que la voix des acteurs y avait été renforcée depuis le début, voir les travaux menés dans le cadre d'ECHO sur la grande salle du Palais de Chaillot à l'époque du TNP, de l'installation laissée par l'ONU en 1951 à la nouvelle sonorisation de 1956. [Lien au dossier pédagogique multimédia en ligne « Entendre le théâtre », à venir début juillet 2019]

21. En 1956, l'installation électroacoustique de Chaillot devait aussi permettre la diffusion d'effets et de décors sonores.

22. Richard Grusin, Premediation: Affect and Mediality After 9/11, Londres et New York, Palgrave Macmillan, 2010.

23. Pour plus de précisions sur ce phénomène, voir « Du média à la médiation : les trente ans de la pensée intermédiale et la résistance théâtrale ", in Jean-Marc Larrue (dir.), Théâtre et intermédialité, Villeneuve d'Ascq, Presses universitaires du Septentrion, 2015, p. 27-56.

24. Il s'agit du "Phelan-Auslander Debate», qui a déchiré le champ anglophone des études théâtrales au tournant des années 2000. Ce débat a connu son paroxysme en 1999 avec la 
publication de Liveness. Performance in a Mediatized Culture de Philip Auslander. Cet ouvrage, qui a eu l'effet d'une bombe, parut quelques mois à peine avant l'autre essai phare de cette période, celui de Bolter et Grusin sur la remédiation dont il partageait les valeurs intermédiales. Auslander y défendait deux idées majeures diamétralement opposées à celles de Phelan: la présence n'est pas l'apanage exclusif du théâtre ; la présence n'est pas, n'a jamais été le contraire du médiatisé et elle n'est pas incompatible avec le médiatisé.

25. Peggy Phelan, Unmarked. The Politics of Performance, Londres et New York, Routledge, 1993, p. 146.

26. Walter Benjamin, L'Euvre d'art à l'époque de sa reproductibilité technique, trad. de l'allemand par Maurice de Gandillac, Paris, Allia, 2003.

27. Gay McAuley, "The Video Documentation of Theatrical Performance », New Theatre Quarterly, vol. 10, n³9, 1994, p.184. Citée par Matthew Reason, Documentation, Disappearance and the Representation of Live Performance, Londres et New York, Palgrave Macmillan, 2014, p. 9.

28. Malcolm Cowley, Writers at Work: The Paris Review Interviews, Londres, Mercury Books, 1962, p. 100. Cité par Matthew Reason, op. cit., p. 10.

29. Matthew Reason, op. cit., p. 21.

30. Ibid.

31. Il s'agit d'un passage choral, très rythmé, presque chanté, que les «belles-sœurs » exécutent parfois en chœur, parfois en solo ou en duo. Elles expriment ainsi leur amour du bingo et décrivent les émotions qu'elles vivent durant une partie.

\section{RÉSUMÉS}

Cet article porte sur l'usage et l'effet de la bande magnétique au théâtre, depuis son apparition dans les années 1950. Il comporte deux parties. L'hypothèse sur laquelle se fonde la première est simple : la bande magnétique audio a radicalement changé l'auralité théâtrale - ce qu'on entend au théâtre, comment on l'entend et ce qu'on en éprouve - et la fabrique du son de la représentation. Ce changement a été déterminant et irréversible. L'article montre que la révolution du son du théâtre est due à la bande magnétique et non aux technologies numériques qui l'ont déclassée.

Mais la bande magnétique audio est aussi, à l'instar des phonogrammes des premiers temps, la précieuse archive d'un moment - l'évènement théâtral - réputé éphémère. Si l'apport de cette archive à la recherche est inestimable, son existence et son statut soulèvent des questions de fond, à la fois sur les pratiques archivistiques et sur la pensée théâtrale, qui font l'objet de la deuxième partie de cet article.

This article examines the use and effect of magnetic tape in theatre since its introduction in the 1950s. It is divided into two parts. The hypothesis on which the first is based is simple: magnetic audiotape has radically changed theatre aurality - what is heard in the theatre and how it is heard and experienced - and the fabric of the sound of the performance. This change has been decisive and irreversible. Our article shows that the revolution in theatre sound is due to magnetic tape, not to the digital technologies that have downgraded it.

Like the early phonograms, however, magnetic audiotape is also the precious archive of a moment - the theatrical event - considered ephemeral. While the contribution of this archive to 
research is invaluable, its existence and status raise fundamental questions about both archival practices and theatrical thought, which are the subjects of the second part of this article.

\section{INDEX}

Mots-clés : auralité, son, théâtre, bande-son, magnétophone, bande magnétique audio, archive Keywords : aurality, sound, theatre, soundtrack, tape recorder, magnetic audio tape, archive

\section{AUTEUR}

\section{JEAN-MARC LARRUE}

Jean-Marc Larrue est professeur de théâtre au département des Littératures de langue française de l'Université de Montréal. Ses recherches portent principalement sur le théâtre du Long $\mathrm{xx}^{\mathrm{e}}$ Siècle (1880 à aujourd'hui) et plus précisément sur le modernisme, les médias et l'intermédialité. Codirecteur du Centre de recherches intermédiales sur les arts, les lettres et les techniques (CRIalt) de 2006 à 2012, il est membre du comité scientifique du Centre de recherche interuniversitaire sur la littérature et la culture québécoises (CRILCQ) depuis cette dernière date. Il a dirigé avec Marie-Madeleine Mervant-Roux le projet de recherche international « Le son du théâtre / Theatre Sound »(2008-2012). Depuis 2014, il est codirecteur, avec Giusy Pisano, du groupe international de recherche « Les arts trompeurs. Machines. Magie. Médias. /

Deceptive Arts. Machines. Magic. Media. »

Parmi ses publications :

Les Archives de la mise en scène. Hypermédialités du théâtre, J.-M. Larrue et G. Pisano (dir.), Lille, Presses universitaires du Septentrion, 2014.

Théâtre et intermédialité, J.-M. Larrue (dir.), Lille, Presses universitaires du Septentrion, 2015. Le Son du théâtre (XIX ${ }^{e}-\mathrm{XXI}^{e}$ siècle). Histoire intermédiale d'un lieu d'écoute moderne, J.-M. Larrue et M.M. Mervant Roux (dir.), Paris, CNRS Éditions, 2016.

Le Triomphe de la scène intermédiale. Théâtre et médias à l'ère électrique, J.-M. Larrue et G. Pisano (dir.), Montréal, Presses de l'Université de Montréal, 2017.

Machines. Magie. Médias, F. Kessler, J.-M. Larrue et G. Pisano (dir.), Villeneuve d'Ascq, Presses universitaires du Septentrion, 2018. 